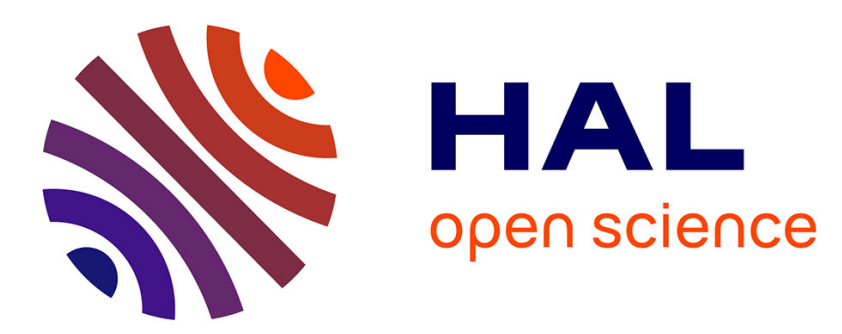

\title{
Anomalous $\mathrm{KCl}(001)$ Surface Corrugation from Fast He Diffraction at Very Grazing Incidence
}

G. a. Bocan, H. Breiss, S. Szilasi, A. Momeni, E. m. Staicu Casagrande, M. s. Gravielle, E. a. Sánchez, Hocine Khemliche

\section{> To cite this version:}

G. a. Bocan, H. Breiss, S. Szilasi, A. Momeni, E. m. Staicu Casagrande, et al.. Anomalous KCl(001) Surface Corrugation from Fast He Diffraction at Very Grazing Incidence. Physical Review Letters, 2020, 125 (9), 10.1103/PhysRevLett.125.096101 . hal-02926236

\section{HAL Id: hal-02926236 \\ https://hal.science/hal-02926236}

Submitted on 18 Dec 2020

HAL is a multi-disciplinary open access archive for the deposit and dissemination of scientific research documents, whether they are published or not. The documents may come from teaching and research institutions in France or abroad, or from public or private research centers.
L'archive ouverte pluridisciplinaire HAL, est destinée au dépôt et à la diffusion de documents scientifiques de niveau recherche, publiés ou non, émanant des établissements d'enseignement et de recherche français ou étrangers, des laboratoires publics ou privés. 


\title{
Anomalous $\mathrm{KCl}(001)$ surface corrugation from fast $\mathrm{He}$ diffraction at very grazing incidence
}

\author{
G. A. Bocan,${ }^{1}$ H. Breiss, ${ }^{2}$ S. Szilasi,${ }^{2}$ A. Momeni, ${ }^{2,3}$ E. M. Staicu \\ Casagrande, ${ }^{2}$ M. S. Gravielle, ${ }^{4}$ E. A. Sánchez, ${ }^{1}$ and H. Khemliche ${ }^{2}$ \\ ${ }^{1}$ Instituto de Nanociencia y Nanotecnología - Nodo Bariloche (CONICET-CNEA) and Instituto Balseiro (U. N. Cuyo), \\ Centro Atómico Bariloche, Av. Bustillo 9500, 8400 S.C. de Bariloche, Argentina \\ ${ }^{2}$ Université Paris-Saclay, CNRS, Institut des Sciences Moléculaires d'Orsay, 91405, Orsay, France \\ ${ }^{3}$ CY Cergy Paris Université, F-95000 Cergy, France \\ ${ }^{4}$ Instituto de Astronomía y Física del Espacio (CONICET-UBA), \\ Casilla de Correo 67, Sucursal 28, C1428EGA Buenos Aires, Argentina
}

\begin{abstract}
We present theoretical and experimental evidence of an anomalous surface corrugation behavior in $\mathrm{He}-\mathrm{KCl}(001)$ for incidence along $\langle 110\rangle$. When the He normal energy decreases below $100 \mathrm{meV}$, i.e. He-surface distances $Z>2 \AA$, the corrugation unexpectedly increases up to an impressive $\gtrsim 85 \%$. This is not due to van der Waals interactions but to the combination of soft potential effects and the evolution of He-cation and He-anion interactions with $Z$. This feature, not previously analyzed on alkali-halide surfaces, may favor the alignement properties of weakly-interacting overlayers.
\end{abstract}

PACS numbers: 34.35.+a, 34.50.-s, 79.20.Rf, 79.60.Bm

The design of promising new materials in the form of thin film stacks brings along the challenge of producing such heterostructures to the highest quality and thus requires an accurate knowledge of the relevant features that rule quasi-epitaxial growth. One such feature is the substrate surface corrugation, which has been shown to drive the overlayer-substrate relative orientation even for weakly-interacting interfaces, such as organic/inorganic or transition metal dichalcogenides systems $[1,2]$.

Grazing incidence fast atom diffraction (GIFAD) [3, 4] is a highly sensitive and non-damaging probe of the projectile-surface interaction potential. Particularly well-suited for a thorough exploration of the surface electronic corrugation, it has already provided valuable structural information on a variety of systems [5-11], and its grazing geometry makes it compatible with in situ monitoring of thin film growth $[12,13]$.

GIFAD occurs when keV-energy projectiles impinge on a surface along a low-index crystallographic direction, under axial surface channeling conditions [14]. The GIFAD pattern arises from the combination of: a) interchannel interference, originated from the periodic array of channels, giving the Bragg peaks and b) intrachannel interference, originated from the corrugation of the interaction potential within a given channel, giving the rainbow peak as well as the supernumerary rainbows [15]. The result on the detection plane is a sequence of Bragg maxima modulated by the underlying intrachannel interference $[15,16]$.

In this work we employ GIFAD to investigate the corrugation of the $\mathrm{KCl}(001)$ surface when probed with low normal energy ${ }^{4} \mathrm{He}^{0}$ projectiles along the $\langle 110\rangle$ channel. We present experimental data, information provided by the potential energy surface (PES) and simulations of the scattering dynamics, all of which put forward a counterintuitive and notorious increase of the surface corrugation with decreasing normal energy $E_{\perp}$ in the low $E_{\perp} \lesssim 60$ $\mathrm{meV}$ range, i.e. for He-surface distances $Z>2.2 \AA$. Although long-distance surface corrugation increases have been experimentally observed by GIFAD for $\mathrm{He} / \mathrm{LiF}(001)$ $(\sim 12 \%) \quad$ [17] and $\mathrm{He} / \mathrm{KBr}(001)(\sim 45 \%) \quad$ [18], this is, to our knowledge, the first time such an overwhelmingly large ( $\gtrsim 85 \%)$ increase is reported. Furthermore, we prove that the ruling factors of this puzzling feature are the evolution of the He-anion and He-cation interactions with $Z$ and the continuance of the projectile-surface interaction during the whole scattering process (i.e. soft potential effects, SPE).

The experiments were performed at room temperature in a GIFAD setup similar to that of Ref. [13]. The $\mathrm{KCl}(001)$ sample was cleaved in air and immediately transferred to the ultra-high-vacuum chamber. Once mounted on a five-axis manipulator it was prepared insitu by annealing at about $650 \mathrm{~K}$ to ensure its cleanness and crystallographic order (corroborated from the well defined GIFAD patterns obtained [19]). The ${ }^{4} \mathrm{He}^{0}$ beam was formed by neutralizing a $\mathrm{He}^{+}$beam with primary energies in the $300-600 \mathrm{eV}$ range. Following the neutralization cell, a set of apertures of $0.4 \times 0.09 \mathrm{~mm}^{2}$ placed $0.36 \mathrm{~m}$ apart were used to reduce the beam divergence to less than $1 \mathrm{mrad}$. The two-dimensional angular distributions of scattered projectiles were collected by placing a detector formed by a Microchannel plate, a phosphor-coated screen and a CCD camera in the forward direction at a distance of $1.27 \mathrm{~m}$. In Figure 1 we depict a GIFAD pattern for $600 \mathrm{eV}^{4} \mathrm{He}$ atoms with $E_{\perp}=21 \mathrm{meV}$. The simulated pattern, that shows good accord with the experiment, was obtained with the Surface Initial Value Representation (SIVR) [20, 21], a wellestablished semi-quantum approach to describe the scattering dynamics [22, 23]. Note that collimation [21], surface defects and thermal vibrations may contribute to 
the elongation of the Bragg spots along the polar angle, though the latter two are not included in the simulations. Such polar spread does not affect our GIFAD-derived results, based on the elastic contribution to the intensity $\left(\theta_{f}=\theta_{i} \pm 0.035^{\circ}\right.$ for the central Bragg order) [19].

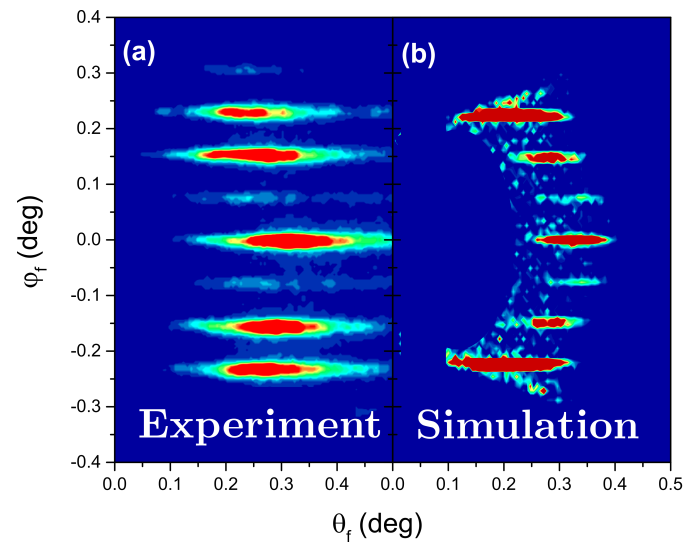

FIG. 1: GIFAD patterns for ${ }^{4} \mathrm{He} \rightarrow\langle 110\rangle \mathrm{KCl}(001)$ with incidence energy $E=600 \mathrm{eV}$ and $E_{\perp}=0.021 \mathrm{eV} \cdot \varphi_{f}\left(\theta_{f}\right)$ : azimuthal(polar) angular position of the scattered atoms.

The experimental corrugation, presented in Fig. 2(a), was obtained by processing the GIFAD patterns to get the effective interaction potential $[7,19,24]$. Since low$E_{\perp}$ scattering takes place far from the surface, it should be ruled by a mild potential landscape, yielding a decreasing surface corrugation with decreasing $E_{\perp}$ but, intriguingly, we find that the corrugation increases with decreasing $E_{\perp}$ and this increase gets particularly sharp below $40 \mathrm{meV}$.

In order to gain more insight into this unexpected behavior we built a high-precision potential energy surface (PES) for the $\mathrm{He} / \mathrm{KCl}(001)$ system based on Density Functional Theory (DFT), using the Perdew-BurkeErnzerhof (PBE) exchange-correlation functional [25] (henceforth the PBE-PES, further details in Ref. [22]). When the PES is averaged along the channeling direction $(\hat{x})$, the axial potential $V^{2 D}(Y, Z)$ that actually rules the scattering process [26] is obtained, where $Y$ is the position across the channelling direction. In Fig. 2(b) we show equipotential curves of the axial PBE-PES, from which the intrinsic corrugation for a given $E_{\perp}$ is obtained from the corresponding amplitude $\Delta Z=Z_{\max }-Z_{\text {min }}$.

Energy regions for which atoms scatter relatively far from the surface $(Z>2 \AA)$ may bring along nonnegligible van der Waals (vdW) interactions, left aside in the PBE-PES. Hence, we built two additional PESs $[27,28]$, where we replaced the PBE exchange-correlation functional by the rVV10 [29] and the DF2b86r [30] ones (henceforth respectively labeled vdW1 and vdW2), which include vdW interactions in a self consistent fashion though with different approaches. The intrinsic corrugations obtained with the three PESs give very similar


FIG. 2: (a) Surface corrugation for ${ }^{4} \mathrm{He} \rightarrow\langle 110\rangle \mathrm{KCl}(001)$ obtained from experiments, the three PESs considered and the respective SIVR simulations. (b) PBE-PES equipotential curves for $0 \leq E_{\perp} \leq 200 \mathrm{meV}$. Inset: Surface geometry.

curves (see Fig. 2(a)), in good accord with the trend of the experimental values, though about $25 \%$ below them. The intrinsic corrugation increase is thus not due to $\mathrm{vdW}$ interactions but should rather be explained through a thorough analysis of the axial PBE-PES [31].

The equipotential profiles across the $\langle 110\rangle$ channel (see inset in Fig. 2(b)) have maxima over the $\mathrm{Cl}^{-}$rows. At mid-channel, over the $\mathrm{K}^{+}$row, there is a central minimum for $E_{\perp} \lesssim 60 \mathrm{meV}$ and a local maximum for $E_{\perp} \gtrsim 60 \mathrm{meV}$. The latter creates two off-center minima which migrate away from mid-channel with increasing $E_{\perp}$. The equipotential curves show that the $\mathrm{He}^{-\mathrm{Cl}^{-}}$ interaction (as obtained from DFT calculations) is always more repulsive than the $\mathrm{He}-\mathrm{K}^{+}$one. The evolution of these interactions upon changing $E_{\perp}$, particularly the slow sinking of the central minimum due to the vanishing He- $\mathrm{K}^{+}$interaction, makes $\mathrm{Z}_{\text {min }}$ vary slower than $\mathrm{Z}_{\text {max }}$, explaining the intrinsic corrugation increase.

The residual discrepancy between the intrinsic and the experimental corrugations remains nevertheless intriguing, leading us to address the rainbow angle [32], a bright peak in the intrachannel contribution at maximal angular deflection $[6,14,15,32]$. The rainbow angle shows a wellknown high sensitivity to the projectile-surface interaction. While the corrugation affects mainly the intensity 
of the specular region to which only trajectories reflecting in the flattest regions of the axial PES contribute, the rainbow probes the steepest regions of the axial PES. For a given $E_{\perp}$, and within a hard-corrugated wall $(\mathrm{HCW})$ approach [33], we determined the PES-rainbow from the steepest slope of the corresponding equipotential curve, while the experimental rainbow was obtained from the processing of the GIFAD pattern [7, 19, 24].

In Fig. 3(a) we show the experimental rainbow angle together with those obtained from the three PESs considered. The increase observed in the experimental rainbow angle for decreasing low $E_{\perp}$ is analogous to the one obtained for the corrugation. The PESs rainbows show a mild increase, associated to the sinking central peak in the potential profiles, but they cannot reproduce the experimental rainbow trend for $E_{\perp}<100 \mathrm{meV}$. The magnitude of the disagreement, once again unrelated to $\mathrm{vdW}$ interactions, strikingly contrasts with the fairly good accord discussed for the corrugation.


FIG. 3: (a) Rainbow angle for ${ }^{4} \mathrm{He} \rightarrow\langle 110\rangle \mathrm{KCl}(001)$. (b) $\mathrm{PBE}$ equipotential curves (Only energies (meV) not shown in Fig. 2(b) are labeled) and trajectories contributing to the rainbow intensity (Dashed black line: HCW, Full black line: SIVR).

Extracting information directly from the $E_{\perp}$ equipotential curves takes into account the projectile-surface interaction only at the turning point (HCW approach), thus neglecting the continuance of the projectile-surface interaction during the rest of the scattering process (i.e. soft potential effects (SPE)). We hence performed simula- tions of the scattering dynamics for each PES considered, by means of the semi-quantum SIVR approach [20, 21] (henceforth labeled PBE-SIVR, vdW1-SIVR and vdW2SIVR). The simulations were performed considering coherent illumination of a single channel (no interchannel interference) so that the rainbow angle can be directly obtained from the diffracted distribution. Strikingly, the PBE-SIVR rainbow reproduces the experiments in the energy range considered with an almost quantitative agreement. Including $\mathrm{vdW}$ contributions results in no significant differences, with vdW1-SIVR and vdW2SIVR running slightly above PBE-SIVR.

The notorious difference observed between the PES and the SIVR rainbow angles, combined with the very good SIVR-Experiment accord, reveal in a quantitative manner the importance of SPE in central aspects of GIFAD. These dynamical effects are naturally included in both the SIVR and the experimental rainbows (obtained by processing the GIFAD patterns) while the PES ones assume unrealistic broken-line trajectories.

Soft potential effects for the PBE-SIVR case can be visualized in Fig. 3(b) where equipotential curves of the axial PES are shown in 1:1 scale. For $3 \lesssim Z \lesssim 5 \AA$ this plot shows a noteworthy shallow $(<7 \mathrm{meV})$ attractive region, due to polarization and lower-order attractive interactions within the DFT PES, which markedly alters the shape and corrugation of the equipotential curves. For high enough $E_{\perp}$ this well is inconsequencial, but low$E_{\perp}$ projectiles traversing this region are strongly affected. This is illustrated in Fig. 3(b) for $E_{\perp}=15 \mathrm{meV}$. Each equipotential curve deviates the trajectory according to the slope at their contact point so that a steeper slope favors $E_{z} \rightarrow E_{y}$ energy conversion. SIVR trajectories deviate from the HCW one upon entering the well region and also during their way out. In the asymptotic condition the projectile-surface interaction fades out and trajectories become linear but not parallel to the HCW trajectory. In the light of this analysis, the difference between the intrinsic and the experimental corrugations could also be due to SPE. Hence, from our SIVR simulations we selected trajectories contributing to the specular intensity. These trajectories are reflected either around the maximum or the minimum of the equipotential, with near-normal incidence and scattering directions. We can compute their SIVR accumulated phase difference (Eq. (10) in Ref. [20]) and obtain an effective corrugation from $\Delta \Phi^{S I V R}=2 k_{\perp}(\Delta Z)^{S I V R}$ (depicted in Fig. 2(a)). The agreement with experiments is remarkable and shows that the PBE-SIVR calculation captures the factors determining the corrugation increase. From Fig. 2(a), the differences between the SIVR and PES curves, together with the SIVR-Experiment accord, provide a quantitative measure of SPE on the effective experimental corrugation obtained by means of the GIFAD technique.

In summary, we report a counter-intuitive increase of the corrugation and the rainbow angle for decreasing low- 
$E_{\perp}$, by means of the GIFAD technique, in ${ }^{4} \mathrm{He}-\mathrm{KCl}(001)$ along the $\langle 110\rangle$ direction. This increase reaches an overwhelmingly large $\gtrsim 85 \%$ and is independently obtained from experiments and simulations. Its origin, unrelated to vdW effects, lies in the different evolution of He-cation and He-anion interactions, combined with SPE. As the latter do not strongly affect the corrugation dependence on $E_{\perp}$, the experimental curve preserves the intrinsic corrugation trend, meaning that GIFAD provides reliable information on this surface feature. In contrast, the rainbow increase is almost fully explained in terms of SPE, strongly enhanced by the shallow well, whose steepest regions markedly deviate low- $E_{\perp}$ rainbow trajectories.

The same two factors are likely responsible for the corrugation increases at low $E_{\perp}$ reported for $\mathrm{He} / \mathrm{KBr}(001)$ [18] and $\mathrm{He} / \mathrm{LiF}(001)$ [17] (and expected for other alkalihalide surfaces) along this same channel. Particularly, for $\mathrm{He} / \mathrm{LiF}$, the much less spectacular corrugation increase is probably only due to SPE as the He- $\mathrm{Li}$ interaction is far vanished in the energy range considered. Also, we expect SPE to rule a marked rainbow increase feature, given the reported presence of shallow wells [34].

Thin alkali-halide layers (mainly $\mathrm{NaCl}$ and $\mathrm{LiF}$ ) are often used to improve charge injection in organic electronic devices. The enhanced corrugation of $\mathrm{KCl}(001)$ at typical physisorption distances may favor the alignment properties of weakly interacting overlayers $[1,2]$, improving their crystalline quality and thus the device performance. The measured corrugation may also be enhanced for probes either more reactive or with larger polarizability such as $\mathrm{H}$ or $\mathrm{Ne}$ [35-37]. Further investigations should address He GIFAD on other alkali-halide surfaces, aiming to maximize the intrinsic corrugation increase.

The authors acknowledge funding from ANPCYT (PICT-2017-1201; PICT-2017-2945) CONICET (PIP 11220130100386 CO; PIP 11220150100274 CO) and U.N. Cuyo (06/C590).

[1] M. Koini, T. Haber, O. Werzer, S. Berkebile, G. Koller, M. Oehzelt, M.G. Ramsey, and R. Resel, Thin Solid Films 517, 483 (2008)

[2] A. Patrykiejew, S. Sokołowski and K. Binder, Surf. Sci. Rep. 37, 207 (2000)

[3] A. Schüller, S. Wethekam and H. Winter, Phys. Rev. Lett. 98, 016103 (2007)

[4] P. Rousseau, H. Khemliche, A. G. Borisov, and P. Roncin, Phys. Rev. Lett. 98, 016104 (2007)

[5] A. Schüller, M. Busch, S. Wethekam, and H. Winter, Phys. Rev. Lett. 102, 017602 (2009)

[6] H. Winter, J. Seifert, D. Blauth, M. Busch, A. Schüller, and S. Wethekam, Appl. Surf. Sci. 256, 365 (2009)

[7] H. Khemliche, P. Rousseau, P. Roncin, V. H. Etgens, and F. Finocchi, Appl. Phys. Lett. 95, 151901 (2009)

[8] B. Lalmi, H. Khemliche, A. Momeni, P. Soulisse, and P. Roncin, J. Phys. Cond. Matt. 24, 442002 (2012)
[9] J. Seifert, M. Busch, E. Meyer, and H. Winter, Phys. Rev. Lett. 111, 137601 (2013)

[10] M. Debiossac, A. Zugarramurdi, H. Khemliche, P. Roncin, A. G. Borisov, A. Momeni, P. Atkinson, M. Eddrief, F. Finocchi, and V. H. Etgens, Phys. Rev. B 90, 155308 (2014)

[11] J. Seifert and H. Winter, Phys. Rev. B 93, 205417 (2016)

[12] P. Atkinson, M. Eddrief, V.H. Etgens, H. Khemliche, M. Debiossac, A. Momeni, M. Mulier, B. Lalmi, and P. Roncin", Appl. Phys. Lett. 105, 021602 (2014)

[13] A. Momeni, E. M. Staicu Casagrande, A. Dechaux, and H. Khemliche, J. Phys. Chem. Lett. 9, 908 (2018)

[14] H. Winter and A. Schüller, Prog. Surf. Sci. 86, 169 (2011)

[15] A. Schüller and H. Winter, Phys. Rev. Lett. 100, 097602 (2008)

[16] A. Schüller, H. Winter, M. S. Gravielle, J. M. Pruneda, and J. E. Miraglia, Phys. Rev. A 80, 062903 (2009)

[17] A. Momeni, P. Soulisse, P. Rousseau, H. Khemliche, and P. Roncin, e-J. Surf. Sci. Nanotech. 8, 101 (2010)

[18] P. Soulisse, PhD thesis, https://tel.archivesouvertes.fr/tel-00625450/, Orsay (2011)

[19] See Supplemental Material at [insert URL] for a set of experimental raw GIFAD patterns and their processing.

[20] M. S. Gravielle and J. E. Miraglia, Phys. Rev. A 90, 052718 (2014)

[21] M. S. Gravielle and J. E. Miraglia, Phys. Rev. A 92, 062709 (2015)

[22] G. A. Bocan and M. S. Gravielle, Nucl. Instrum. Meth. Phys. Res. B 421, 1 (2018)

[23] L. Frisco, J. E. Miraglia and M. S. Gravielle, J. Phys. Condens. Matter 30405001 (2018)

[24] A. Schüller, M. Busch, J. Seifert, S. Wethekam, H. Winter, and K. Gärtner, Phys. Rev. B 79, 235425 (2009)

[25] J. P. Perdew, K. Burke and M. Ernzerhof, Phys. Rev. Lett. 77, 3865 (1996)

[26] A. Zugarramurdi and A. G. Borisov, Phys. Rev. A 86, 062903 (2012)

[27] See Supplemental Material at [insert URL] for vdW-PESs info.

[28] P. Giannozzi et al., J. Phys.: Cond. Matt. 21, 395502 (2009)

[29] R. Sabatini, T. Gorni and S. de Gironcoli, Phys. Rev. B 87, 041108(R) (2013)

[30] I. Hamada, Phys. Rev. B 89, 121103(R) (2014)

[31] M. del Cueto, R. J. Maurer, A. Al Taleb, D. Farías, F. Martín, and C. Díaz, J. Phys. Cond. Matt. 31, 135901 (2019)

[32] U. Specht, M. Busch, J. Seifert, H. Winter, K. Gärtner, R. Włodarczyk, M. Sierka, and J. Sauer, Nucl. Instrum. Meth. Phys. Res. B 269, 799 (2011)

[33] U. Garibaldi, A. C. Levi, R. Spadacini, and G. E. Tommei, Surf. Sci. 48, 649 (1975)

[34] M. Debiossac, A. Zugarramurdi, P. Lunca-Popa, A. Momeni, H. Khemliche, A. G. Borisov, and P. Roncin, Phys. Rev. Lett. 112, 023203 (2014)

[35] D. Farías, H.F. Busnengo and F.Martín, J. Phys. Cond. Matt. 19, 305003 (2007)

[36] M.S. Gravielle, A. Schüller, H. Winter, and J.E. Miraglia, Nucl. Instr. \& Meths. Phys. Res. B 269, 1208 (2011)

[37] M. Minniti, C. Díaz, J.L. Fernández Cuñado, A. Politano, D. Maccariello, F. Martín, D. Farías and R. Miranda, J. Phys. Cond. Matt. 24, 354002 (2012) 